Published in final edited form as:

Exp Eye Res. 2010 February ; 90(2): 210. doi:10.1016/j.exer.2009.10.007.

\title{
The Presence and Distribution of Elastin in the Posterior and Retrobulbar Regions of the Mouse Eye
}

\author{
Scott Gelman, Frances E. Cone, Mary E. Pease, Thao D. Nguyen, Kristin Myers, and Harry A. \\ Quigley \\ Glaucoma Research Laboratory, Wilmer Ophthalmological Institute, Johns Hopkins University \\ School of Medicine, Baltimore, MD USA.
}

\begin{abstract}
The Presence and distribution of elastin in the posterior and retrobulbar regions of the mouse eye was investigated. Mice of two strains (C57/BL6 and DBA/2J) were studied at 2 months and 8-12 months of age. Light, confocal, and transmission electron microscopy were used to identify elastin, using immunohistochemical techniques and ultrastructural evaluation. Elastin was found in the following ocular structures: conjunctiva, muscle tendons, sclera, choroid, and meninges. The elastin in the sclera was most dense in a ring surrounding the peripapillary optic nerve head, with its presence in the inner sclera declining with greater distance from the nerve head. Elastin fibers were oriented in the sclera along what would be expected to be the principal stress directions generated from the intraocular pressure, though actual biomechanical measurements have not yet been made in the mouse sclera. Elastin comprises a portion of the mouse sclera and its distribution in the peripapillary area is similar to that in human eyes.
\end{abstract}

\section{Keywords}

elastin; mouse; sclera; optic nerve; fibrillin; glaucoma

\section{Introduction}

The internal structures of the eye are enclosed within the corneoscleral shell, made up of connective tissues that serve as both the primary refracting surface and load-bearing structure. The sclera has a central role in maintaining the mechanical integrity of the pressurized eye. Changes in intraocular pressure (IOP) cause scleral deformation that is transmitted to the optic nerve head, the site of glaucoma damage (Quigley et al., 1981), causing potentially damaging deformation (Sigal et al., 2005). An alteration in the structure or the material properties of the sclera will affect the deformation response to a given level of IOP. IOP and ocular blood flow provide both constant and pulsating stress in the eyewall that must be tolerated without permanent damage to its principal elements: collagen, elastin, and glycosaminoglycans.

\footnotetext{
(C) 2009 Elsevier Ltd. All rights reserved.

Correspondence: Scott Gelman, The Smith Building, Room 002, 400 North Broadway, Baltimore MD 21287. sgelman2@ jhmi.edu Phone: 410-955-3777 Fax: 443-287-2711.

Publisher's Disclaimer: This is a PDF file of an unedited manuscript that has been accepted for publication. As a service to our customers we are providing this early version of the manuscript. The manuscript will undergo copyediting, typesetting, and review of the resulting proof before it is published in its final citable form. Please note that during the production process errors may be discovered which could affect the content, and all legal disclaimers that apply to the journal pertain.
} 
Collagens I and III impart inherent tensile strength to the human sclera, complemented by elastin, which permits elastic deformation and recovery (Kielty et al, 2002). This function is critical to the maintenance of scleral integrity given the repeated cycles of loading produced by the choroidal vessels (Faury, 2001). Elastin is a complex of deposited tropoelastin on a template of fibrillin-rich microfibrils (Mecham et al., 1994). Tropoelastin is a $60-70 \mathrm{kDa}$ protein composed of alternating hydrophobic and lysine-containing cross-linking domains (Gray et al., 1973). Of approximately 40 lysine residues in the secreted tropoelastin monomer, many are cross-linked by lysyl oxidase producing great stability and insolubility of the protein. Elastin has a typical longevity equal to the human lifespan (Shapiro et al., 1991) and its multistep assembly pattern is tissue-specific, indicating that renovation or replacement may be difficult in adult tissues (Wagensweil and Mecham, 2007). Mice lacking both alleles of the one elastin gene die at or before birth, while those with one normal allele live a short time, but have defective arteries with increased numbers of elastic lamellae (Dietz and Mecham, 2000).

The microfibrils surrounding the elastin core may participate in extensibility of the complex (Wang et al., 2009). Fibrillin interacts with transforming growth factor $\beta$, regulating its activation, and abnormality in this function is related to development of Marfan syndrome (Neptune et al., 2003). Fibrillin-containing microfilaments are also found without elastin in the lens zonules.

Alterations in elastin may be either a result of glaucomatous damage to the eye or may participate in its causation. Hernandez and colleagues detected changes in elastin in human glaucoma eyes (Hernandez et al., 1989, 1992; Pena et al., 1998). In studies of both human and monkey eyes with glaucoma damage, our laboratory found an altered appearance of elastin without actual loss of fibers (Quigley, H.A., 1991a, 1991b, 1994; Quigley, E.N., 1996). We speculated that elastin was seemingly disconnected from the remainder of the scleral and optic nerve head connective tissue matrix. Since African-derived persons have a greater prevalence of glaucoma compared to European-derived persons, it is interesting that they exhibit differences in elastin appearance in the optic nerve head region (Urban et al., 2007). Intriguingly, polymorphisms in the lysyl oxidase-like 1 gene are associated with exfoliation syndrome, the most common syndrome associated with open angle glaucoma (Thorleifsson et al., 2007). The protein coded by this gene participates in modification of elastin.

Animal models of glaucoma have added important information to our understanding of its pathogenesis, with recent use of both spontaneous (Jakobs et al., 2005) and induced (Grozdanic et al., 2003) mouse models of glaucoma. The relevance of mouse research in glaucoma depends upon the degree of homology of important ocular structures to the human eye. We did not find previous descriptions of the detailed structure of the sclera in the mouse, nor any prior mention of the presence or distribution of murine ocular elastin. Given its relevance to the biomechanical response of the eye to IOP, the present report presents the first description of the distribution of elastin in mouse sclera.

\section{Methods}

\section{Animals Used}

A total of 41 mice of two strains were used: $10 \mathrm{C} 57 / \mathrm{B} 16$ at 8 weeks of age, $10 \mathrm{C} 57 / \mathrm{B} 16$ at 48 weeks of age, $10 \mathrm{DBA} / 2 \mathrm{~J}$ at 8 weeks of age and $11 \mathrm{DBA} / 2 \mathrm{~J}$ at 56 weeks of age. All animals were treated in accordance with the ARVO Statement for Use of Animals in Ophthalmic and Vision Research using protocols approved and monitored by the Animal Care Committee of the Johns Hopkins University School of Medicine. Animals were housed with a $14 \mathrm{~h}$ light/10 $\mathrm{h}$ dark cycle with standard chow and water ad libitum. 


\section{Sacrifice and Tissue Processing}

Animals were anesthetized with a mixture of ketamine, xylazine, and acepromazine at 50, 10 and $2 \mathrm{mg} / \mathrm{kg}$, respectively, and both eyes were enucleated. Prior to fixation, right eyes were manually inflated to their normal IOP $(\sim 15 \mathrm{~mm} \mathrm{Hg})$, then their axial lengths were measured with a digital caliper (Instant Read-Out Digital Caliper, Electron Microscopy Sciences, Hatfield, PA). Left eye optic nerve and the entire right eye were fixed by immersion in $4 \%$ paraformaldehyde in $0.1 \mathrm{M}$ phosphate buffer (pH 7.2) and post-fixed in $1 \%$ osmium oxide in $0.1 \mathrm{M}$ phosphate buffer ( $\mathrm{pH} 7.2$ ) for $2 \mathrm{hrs}$. The right eyes were divided into 4 distinct longitudinal portions (temporal, superior, nasal \& inferior) containing sclera, choroid and retina, along with a circular retrobulbar sample (centered on the optic disc). These were then processed for transmission electron microscopy (TEM) by dehydration in graded alcohol, exposure to $1.0 \%$ uranyl acetate in $100 \%$ ethanol for 1 hour and embedded in epoxy resin.

The left eyes, after optic nerve removal, were inflated to $15 \mathrm{~mm} \mathrm{Hg}$ by insertion of a needle into the posterior chamber connected to a reservoir, containing 2\% paraformaldehyde (PFA) in 5\% sucrose in $0.1 \mathrm{M}$ phosphate buffer ( $\mathrm{pH} 7.2)$, whose height set the pressure. The eye was then fixed by immersion in 2\% paraformaldehyde (PFA) in 5\% sucrose in $0.1 \mathrm{M}$ phosphate buffer ( $\mathrm{pH}$ 7.2) for one hour. Eyes were taken through graded sucrose solutions, 5\%, 10\%, $12.5 \%$, and $15 \%$, for 30 minutes each, and then placed in $20 \%$ sucrose overnight. After 30 minutes of 2:1 $20 \%$ sucrose solution to optimal cutting temperature (OCT) compound (Sakura Finetek USA, Torrance, CA), eyes were embedded in fresh sucrose-OCT. Cryosections, $8 \mu \mathrm{m}$ thick, were collected from the peripapillary region onto slides (SuperFrost Plus; Fisher Scientific, Pittsburgh, PA) and stored at $-80^{\circ} \mathrm{C}$ before immunolabeling.

\section{Histochemistry}

The primary antibody was rabbit anti-elastin TP592 (Elastin Products Company, Owensville, MO) at a dilution of 1:250. Sections were washed in Tris-buffered saline (TBS) containing $0.1 \%$ Triton X-100 for 10 minutes, dehydrated in 3\% hydrogen peroxide for 30 minutes and again washed in TBS $3 \times 10$ minutes. Non-specific binding sites were blocked with $2 \%$ normal goat serum in TBS for $1 \mathrm{hr}$ followed by a 10 minute TBS wash. Avidin and biotin blocking kit (Vector Laboratories, Burlingame, CA) was used before incubation of primary antibody in $1 \%$ BSA/TBST overnight at $4^{\circ} \mathrm{C}$. After washing in TBS $(3 \times 10$ minutes $)$, sections were incubated in biotin-labeled goat anti-rabbit secondary antibody (Kirkegaard \& Perry Laboratories, Inc., Gaithersburg, MD) at 1:500 in 1\% BSA/TBS for 1 hour. Slides were washed and incubated with the AEC Substrate Kit (Vector Laboratories, Burlingame, CA). Negative control experiments included non-immune serum of the same species as the primary antibody at the same protein concentration in incubation buffer alone. Labeled sections were mounted with VectaMount AQ Mounting Medium (Vector Laboratories, Burlingame, CA). Images of all slides were captured digitally using standardized microscope and camera settings (Axioskop and Axiocam with Axiovision ver. 3 software; Carl Zeiss, Thornwood, NY).

For identification of elastin in the peripapillary region by the Luna method, cryosections were treated with serial exposure to $95 \%$ ethanol for 3 minutes, aldehyde-fuchsin solution $(0.5 \%$ basic fuchsin in $1.5 \%$ acetaldehyde in $1 \% 12 \mathrm{~N} \mathrm{HCl}$ in $70 \%$ ethanol) for 40 minutes, four 1 minute rinses of $95 \%$ ethanol, two 3 minute washes of $100 \%$ ethanol and two 3 minute washes of xylene. Sections were coverslipped with Permount for observation under bright field light microscopy (Zeiss Axioskop).

\section{Transmission Electron Microscopy}

Scleral and peripapillary epoxy-embedded tissues were sectioned at 1 micron thickness for orientation, then ultrathin sections taken at representative areas. Peripapillary specimens were sectioned toward the optic nerve head from the superior side until the immediate zone around 
and including the nerve head was reached. Thin sections were stained by a $0.01 \%$ uranyl acetate and $0.57 \%$ tannic acid solution followed by the addition of $0.3 \%$ Reynolds lead citrate solution before examination in the transmission electron microscope (Hitachi H7600; Hitachi HighTechnologies Corp., Tokyo, Japan).

\section{Results}

Elastin was found in the sclera as well as in the choroid, conjunctiva, muscle tendons, and meninges of the normal mouse eye. The specificity of the Luna stain technique for identification of elastin was confirmed by staining of serial sections of optic nerve head and normal aorta in cross section with both anti-elastin antibody and Luna (FIGURE 1). There was elastin present in the sclera, varying in quantity depending upon the proximity to the optic nerve head and to the choroid. Elastin fibers were most dense in the peripapillary area, configured in a ring surrounding the optic nerve head and consisting of a group of 10-12 elastin fibers running in parallel (FIGURE 2). The high density of elastin in the peripapillary region was confirmed by tranmission electron microscopy.

There was no elastin or other connective tissue elements within the optic nerve head itself. The amount of scleral elastin decreased with increasing distance away from the optic nerve head. By $1 \mathrm{~mm}$ from the nerve head, the amount had fallen to levels not detectable by light microscopy. Elastin fibers most often appeared to have a straight profile in the sclera. While their orientation was parallel to the scleral surface, it had no preferred direction once away from the peripapillary area (FIGURE 3 and FIGURE 4). Outside the immediate peripapillary area, elastin was found only in the innermost sclera, adjacent to the choroid (FIGURE 5).

By TEM, elastin fibers and their associated microfibrillar matrix were similar in appearance to those in human eyes. They were typically closely adjacent to fibrillar collagen in the sclera. In the choroid outside the immediate peripapillary area, elastin was present in the central layer of Bruch's membrane and at the junctional zone between the choroid and sclera, but not in the middle and outer sclera. There were infrequent fibers of elastin within the mid-choroid.

In the conjunctiva, elastin fibrils were oriented in varying directions. A similar pattern of varying orientation of elastin was also present in the dura mater (FIGURE 6). The pia mater had a more specialized orientation of elastin consisting of longitudinally oriented fibers immediately adjacent to the optic nerve, encased by an outer circumferential ring of elastin. Elastin was found in extraocular muscle tendons (data not shown). There were no qualitative differences in elastin between younger and older mouse eyes of either strain. Alterations seen in older DBA/2J mice, after the occurrence of spontaneous glaucoma, will be reported separately.

\section{Discussion}

To our knowledge, this is the first description of the presence and configuration of elastin in the normal murine eye. We found that mouse sclera has a similar distribution to that in human eyes. Both species have dense collections of elastin circumferentially oriented around the optic nerve head. Extensive biomechanical literature, using both modeling techniques (Sigal, 2005) and in vivo studies (Burgoyne, 2005; Downs, 2003, 2005) suggests that the IOP generates significant tensile stresses distributed circumferentially and meridionally within the plane of the sclera. The circumferential stresses are magnified at the transition between the stiffer sclera and more compliant optic nerve head. As in human eyes, elastin in murine eyes is oriented to reinforce the weaker optic nerve head opening and resist circumferential expansion induced by the IOP-generated scleral wall stress. This elastin ring provides a mechanism for reversible expansion and contraction when variations in IOP occur. 
In addition to elastin, the extracellular matrix of the sclera is composed of a network of crosslinked collagen and proteoglycans. The complex interplay of these extracellular matrix products provides strength and flexibility to withstand wall stress and to prevent optic nerve head damage. Though elastin shows some regenerative ability in skin, its very low turnover rate suggests that it is designed to last many decades, though degeneration does occur in arterial elastin over a lifetime of deformation and recovery (O'Rourke, 2007). Past research (cited above) indicates that changes in elastin and the surrounding connective tissues are related to glaucoma's pathogenesis. The loss of normal elastin function could cause the inability of the tissue to recover from deformation thus causing an alteration in peripapillary dimensions. Damage to the elastin network could compromise the tissue's mechanical stiffness to resist initial deformations. We plan to study the effects of spontaneous and induced glaucoma damage in mice to determine their biomechanical and histopathological effects on the sclera.

We observed that the orientation of elastin fibers in the tissues of the eye correspond to the directions of typical mechanical stresses. The conjunctiva and the dura mater are subject to motion in a variety of directions. In the case of the dura, the known variation in the pressure within the subarachnoid space must be accommodated by expansion and contraction over short time periods. It is not surprising that elastin is distributed in a relatively random direction in this tissue. The pia mater is closely adherent to the optic nerve. While the nerve axons can swell in some conditions, there is a need to resist elongation of the nerve as well as to accommodate the torsion in the nerve as the eye undergoes excursions generated by the extraocular muscles. Hence, the pia has two layers of elastin, one running circumferentially and one parallel to the long axis of the nerve.

It is likely that the elastin in the sclera away from the nerve head and within the choroid may provide further suggestions about the mechanical stress state in these tissues. From biomechanical considerations, the stress across the sclera would be greatest at its inner portion and least at the outer surface. Thus, it is logical that the inner sclera would be invested with more elastin. As to elastin in the choroid, the central component of Bruch's membrane has long been known to contain elastin. While the other 4 layers of Bruch's membrane chiefly consist of collagens, its elastin may provide flexible recoil and avoid rupture, which is a known antecedent to various chorioretinopathies. The presence of elastin within the extracellular space of the choroid may provide an elasticity that permits expansion and contraction.

The elastin of the mouse eye is configured similarly to that of human eyes and provides further opportunity to learn about the effects of glaucoma on this connective tissue component in murine models.

\section{Acknowledgments}

Supported in part by PHS Research Grants EY 02120 (Dr Quigley) and 01765 (Wilmer Institute Core Grant), and by the Leonard Wagner Trust, New York, NY and by unrestricted support from William T. Forrester.

\section{Reference}

Burgoyne CF, Downs JC, Bellezza AJ, Suh JK, Hart RT. The optic nerve head as a biomechanical structure: a new paradigm for understanding the role of IOP-related stress and strain in the pathophysiology of glaucomatous optic nerve head damage. Prog. Retin. Eye Res 2005;24:39-73. [PubMed: 15555526]

Dietz HC, Mecham RP. Mouse models of genetic diseases resulting from mutations in elastic fiber proteins. Matrix Biology 2000;19:481-488. [PubMed: 11068202]

Downs JC, Suh JK, Thomas KA, Bellezza AJ, Burgoyne CF, Hart RT. Viscoelastic characterization of peripapillary sclera: material properties by quadrant in rabbit and monkey eyes. J. Biomech. Eng 2003;125:124-131. [PubMed: 12661206] 
Downs JC, Suh JK, Thomas KA, Bellezza AJ, Hart RT, Burgoyne CF. Viscoelastic material properties of the peripapillary sclera in normal and early-glaucoma monkey eyes. Invest. Ophthalmol. Vis. Sci 2005;46:540-546. [PubMed: 15671280]

Faury G. Function-structure relationship of elastic arteries in evolution: from microfibrils to elastin and elastic fibres. Pathol. Biol 2001;49:310-325. [PubMed: 11428167]

Gray WR, Sandberg LB, Foster JA. Molecular model for elastin structure and function. Nature 1973;246:461-466. [PubMed: 4762195]

Grozdanic D, Betts DM, Sakaguchi DS, Allbaugh RA, Kwon YH, Kardon RH. Laser-induced mouse model of chronic ocular hypertension. Invest. Ophthalmol. Vis. Sci 2003;44:4337-46. [PubMed: 14507878]

Hernandez MR, Luo XX, Andrzejewska W, Neufeld AH. Age-related changes in the extracellular matrix of the human optic nerve head. Am. J. Ophthalmol 1989;107:476-484. [PubMed: 2653045]

Hernandez MR. Ultrastructural immunocytochemical analysis of elastin in the human lamina cribrosa. Changes in elastic fibers in primary open-angle glaucoma. Invest. Ophthalmol. Vis. Sci 1992;33:28912903. [PubMed: 1526740]

Jakobs TC, Libby RT, Ben Y, John SW, Masland RH. Retinal ganglion cell degeneration is topological but not cell type specific in DBA/2J mice. J. Cell Biol 2005;171:313-25. [PubMed: 16247030]

Kielty CM, Sheratt MJ, Shuttleworth CA. Elastic fibres. J. Cell Sci 2002;115:2817-2828. [PubMed: 12082143]

Mecham, RP.; Davis, EC. Elastic fiber structure and assembly. In: Yurchenco, PD.; Birk, DE.; Mecham, RP., editors. Extracellular Matrix Assembly and Structure. Academic Press; New York: 1994. p. 281-314.

Neptune ER, Frischmeyer PA, Arking DE, Myers L, Bunton TE, Gayraud B, Francesco R, Sakai LY, Dietz HC. Dysregulation of TGF-beta activation contributes to pathogenesis in Marfan syndrome. Nat. Genet 2003;33:407-411. [PubMed: 12598898]

O'Rourke MF. Arterial aging: pathophysiological principles. Vasc. Med 2007;12:329-341. [PubMed: 18048471]

Pena JD, Netland PA, Vidal I, Dorr DA, Rasky A, Hernandez MR. Elastosis of the lamina cribrosa in glaucomatous optic neuropathy. Exp. Eye. Res 1998;67:517-524. [PubMed: 9878213]

Quigley EN, Quigley HA, Pease ME, Kerrigan LA. Quantitative studies of elastin in the optic nerve heads of persons with open-angle glaucoma. Ophthalmology 1996;103:1680-5. [PubMed: 8874442]

Quigley HA, Addicks EM, Green WR, Maumenee AE. Optic nerve damage in human glaucoma. II. The site of injury and susceptibility to damage. Arch. Ophthalmol 1981;99:635-49. [PubMed: 6164357]

Quigley HA, Brown A, Dorman-Pease ME. Alterations in elastin of the optic nerve head in human and experimental glaucoma. Br. J. Ophthalmol 1991a;75:552-7. [PubMed: 1911659]

Quigley HA, Dorman-Pease ME, Brown AE. Quantitative study of collagen and elastin of the optic nerve head and sclera in human and experimental monkey glaucoma. Curr. Eye. Res 1991b;10:877-88. [PubMed: 1790718]

Quigley HA, Pease ME, Thibault D. Change in the appearance of elastin in the lamina cribrosa of glaucomatous optic nerve heads. Graefe's Arch. Clin. Exp. Ophthalmol 1994;232:257-61. [PubMed: 8045433]

Shapiro SD, Endicott SK, Province MA, Pierce JA, Campbell EJ. Marked longevity of human lung parenchymal elastic fibers deduced from prevalence of D-aspartate and nuclear weapons-related radiocarbon. J. Clin. Invest 1991;87:1828-1834. [PubMed: 2022748]

Sigal IA, Flanagan JG, Ethier CR. Factors influencing optic nerve head biomechanics. Invest. Ophthalmol. Vis. Sci 2005;46:4189-4199. [PubMed: 16249498]

Thorleifsson G, Magnusson KP, Sulem P, Walters GB, Gudbjartsson DF, Stefansson H, Jonsson T, Jonasdottir A, Jonasdottir A, Stefansdottir G, Masson G, Hardarson GA, Petursson H, Arnarsson A, Motallebipour M, Wallerman O, Wadelius C, Gulcher JR, Thorsteinsdottir U, Kong A, Jonasson F, Stefansson K. Common sequence variants in the LOXL1 gene confer susceptibility to exfoliation glaucoma. Science 2007;317:1397-400. [PubMed: 17690259]

Urban Z, Agapova O, Hucthagowder V, Yang P, Starcher BC, Hernandez MR. Population differences in elastin maturation in optic nerve head tissue and astrocytes. Invest. Ophthalmol. Vis. Sci 2007;48:3209-3215. [PubMed: 17591890] 
Wagensweil JE, Mecham RP. New insights into elastic fiber assembly. Birth Defects Research (Part C) 2007;81:229-240.

Wang MC, Lu Y, Baldock C. Fibrillin microfibrils: a key role for the interbead region in elasticity. J. Mol. Biol 2009;388:168-79. [PubMed: 19268673] 


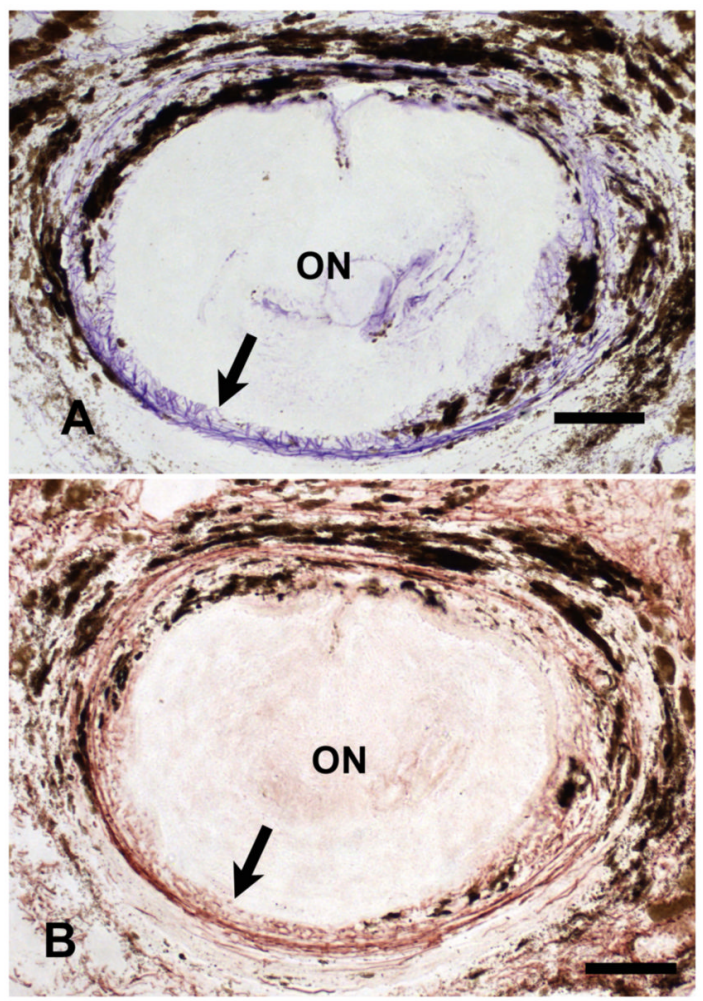

Figure 1.

Peripapillary sclera of young C57/BL6 mouse in cross-section. The upper micrograph shows a dense ring of purple, Luna-stained elastin fibers (arrow) is present immediately adjacent to the optic nerve head. A serial section from the same sample (bottom) that is stained with rabbit anti-elastin primary antibody demonstrates that the material stained by the Luna technique is similar in configuration to that identified by anti-elastin (arrow). (Top x20, bar $=40 \mathrm{um}$; bottom $\mathrm{x} 20$, bar $=50 \mathrm{um}$ ) 

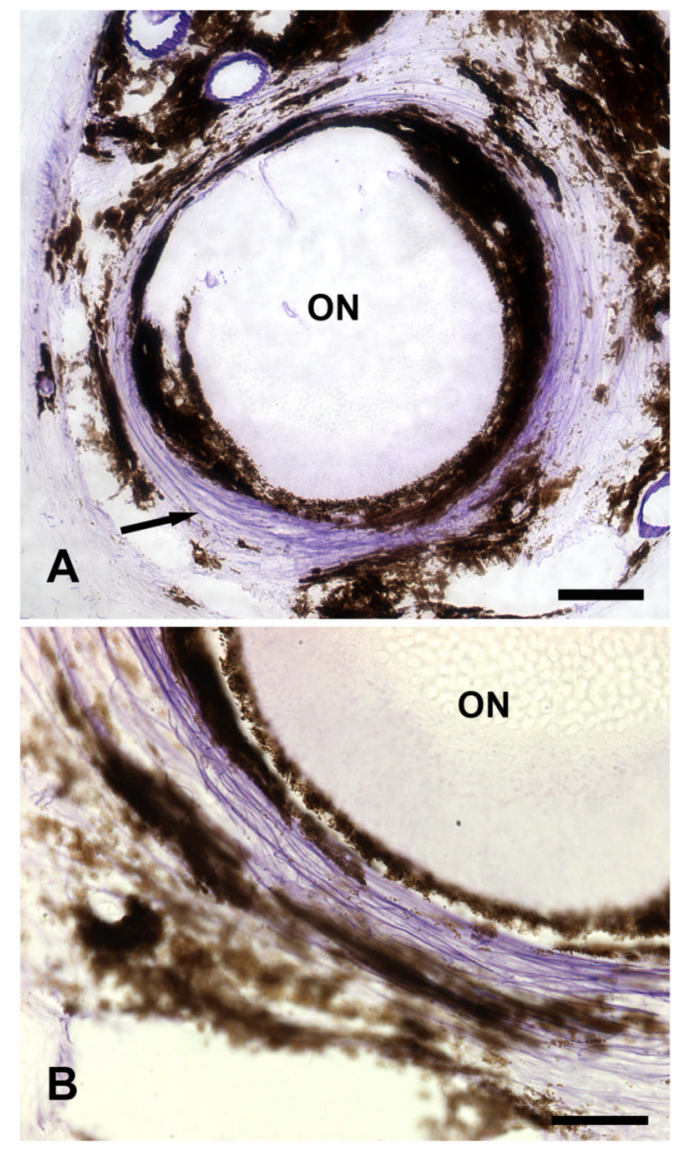

Figure 2.

Peripapillary sclera of young C57/BL6 mouse in cross-section. A dense ring of purple, Lunastained elastin fibers is present in a circumferential orientation (arrow) immediately adjacent to the optic nerve head. The upper micrograph shows a complete cross section with the position of the optic nerve $(\mathrm{ON})$ in the center. A magnified region of the ring (bottom) demonstrates the purple-stained fibers running together in the sclera with dark, melanin-containing choroidal cells flanking them. (Top x20, bar $=40 \mathrm{um}$; bottom x63, bar $=30 \mathrm{um}$ ) 

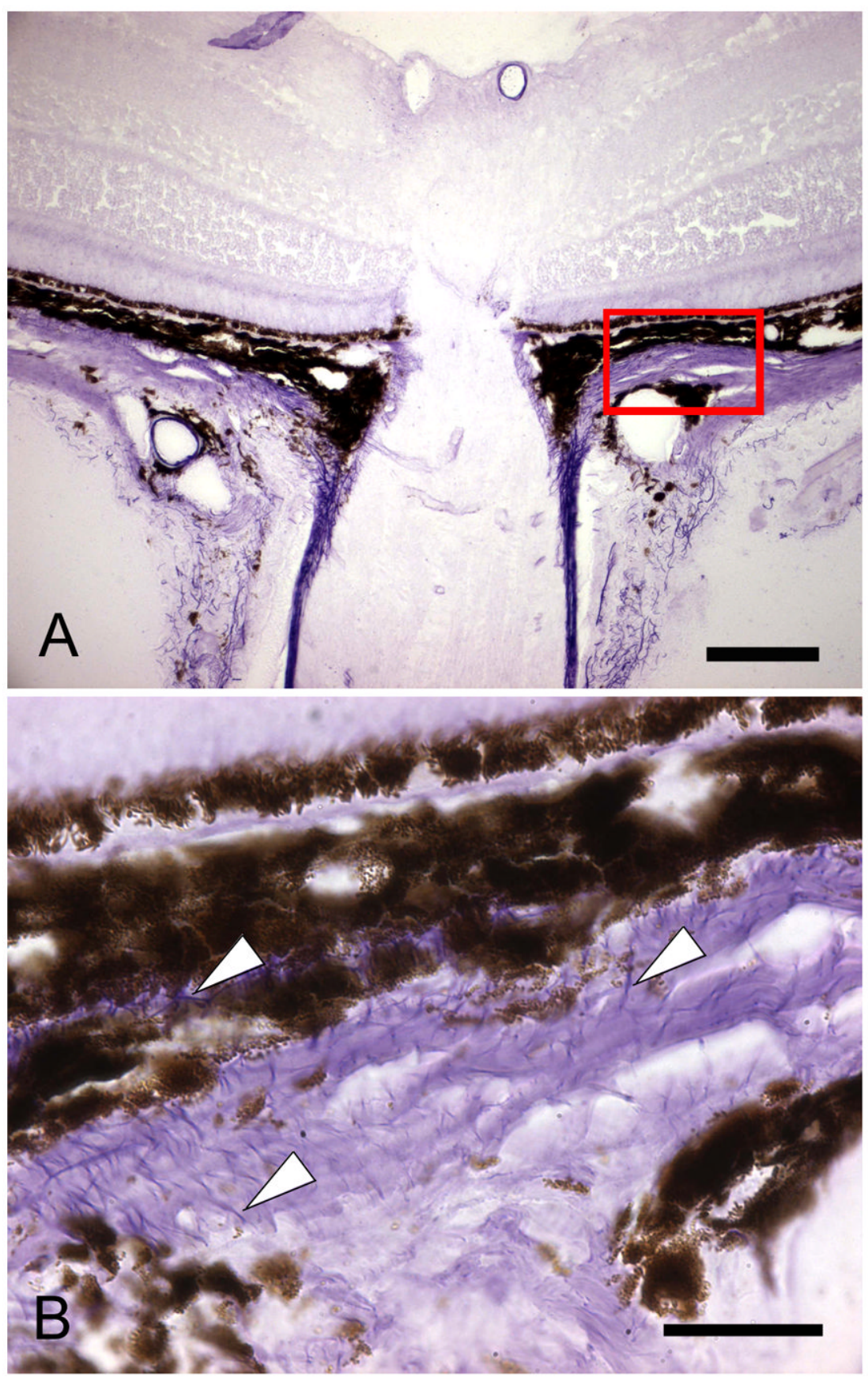

Figure 3.

The upper light micrograph (x20, bar=40um) demonstrates the location of the magnified image (bottom) of sclera and choroid near the optic nerve head and extending away from it (to right of section). Luna-stained elastin (arrowheads) is dense near the nerve head (lower left) and is found throughout the sclera in this region, but with no preferred orientation away from the nerve. (C57/BL6 mouse eye, Luna method, frozen section, X100, bar =10 um bar). 


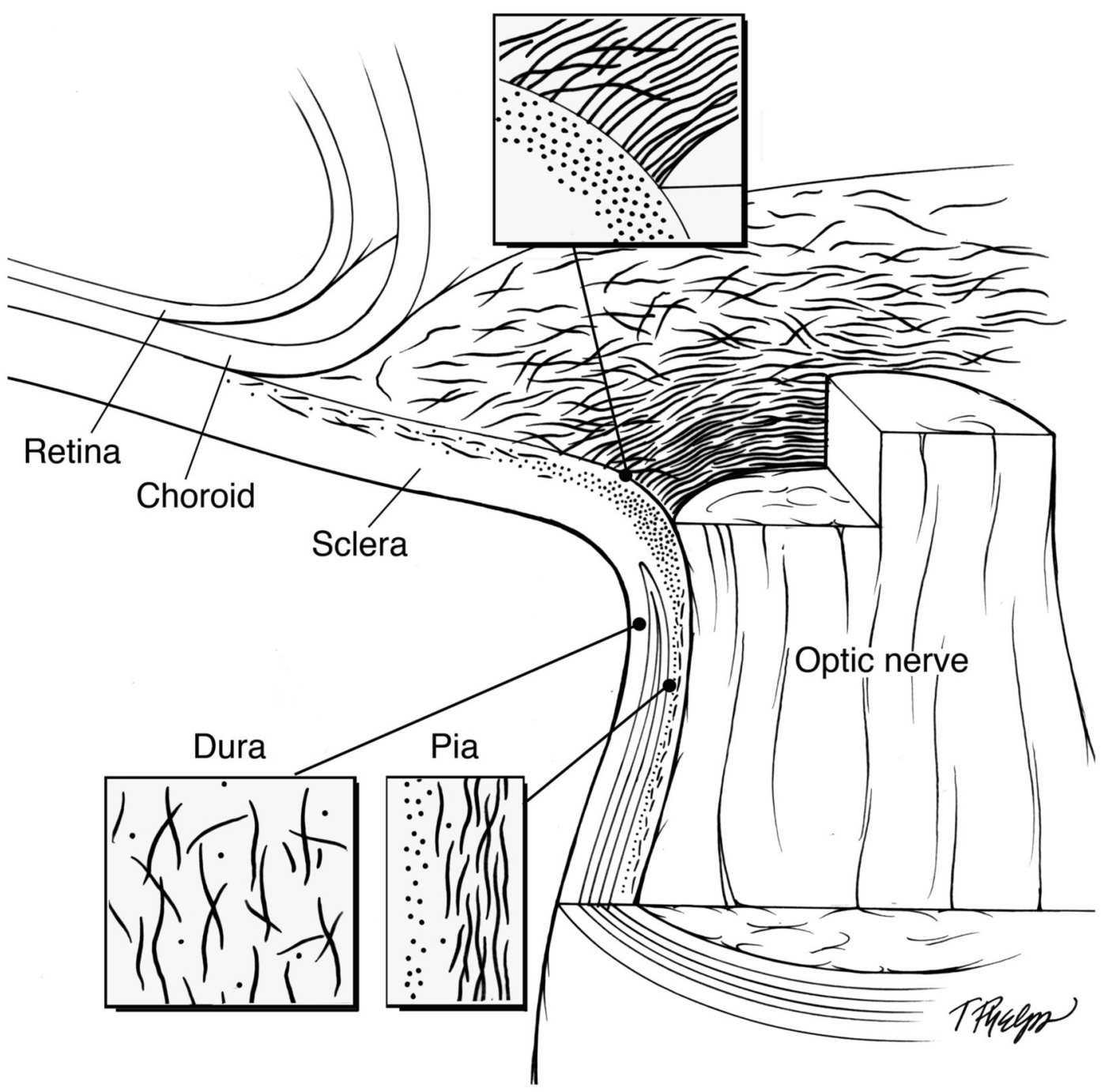

Figure 4.

Schematic illustration showing the elastin distribution and configuration. The optic nerve head, dura mater and pia mater are each magnified to show the region specific orientation of elastin. Relevant quantities of elastin fibers are depicted to demonstrate the difference of distribution in the sclera as it relates to the distance away from the optic nerve head. 


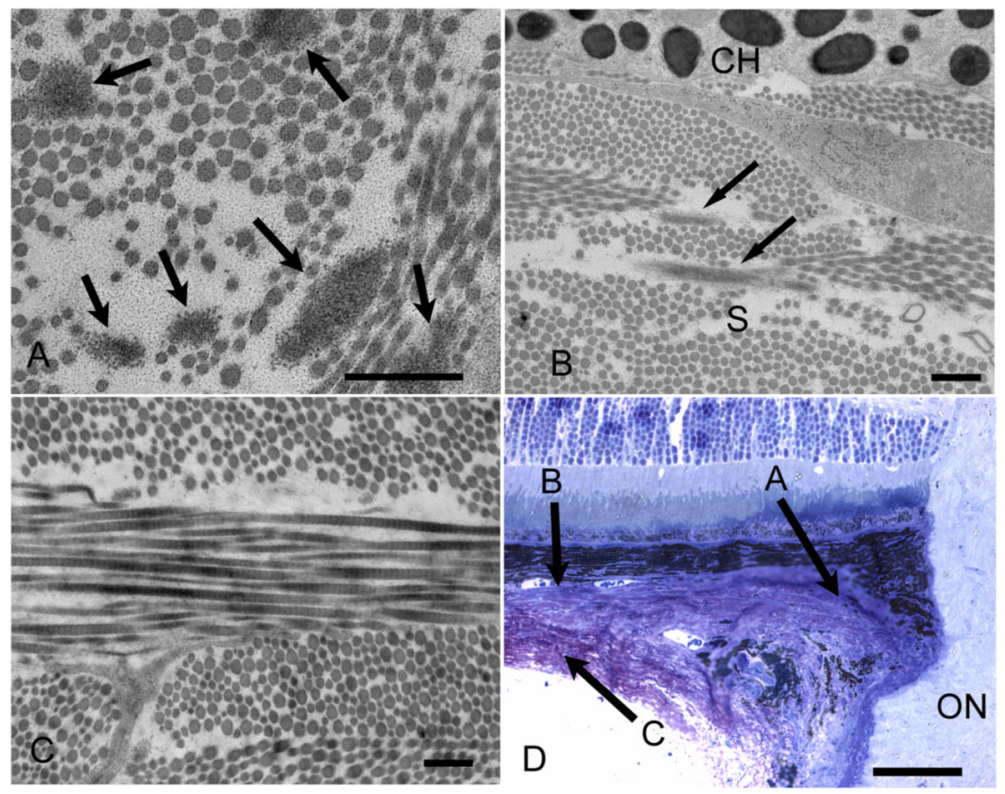

Figure 5.

Transmission electron micrograph of C57/BL6 mouse sclera in cross-section. A. Six elastin fibers (arrows) are present in the peripapillary sclera, the region where elastin is most dense $(\mathrm{x} 12,000$, bar $=500 \mathrm{~nm})$. B. Elastin is present (arrows) in the innermost sclera adjacent to the choroid, whose melanocytes are seen at top $(x 10,000$, bar $=500 \mathrm{~nm})$. C. Outer sclera region showing no elastin present $(\mathrm{x} 10,000$, bar=500nm). D Light micrograph demonstrating the regions that each of the transmission electron micrographs is representing ( $\mathrm{x} 40, \mathrm{bar}=50 \mathrm{um})$. Variable diameter collagen fibrils are seen throughout the sclera in all images. 


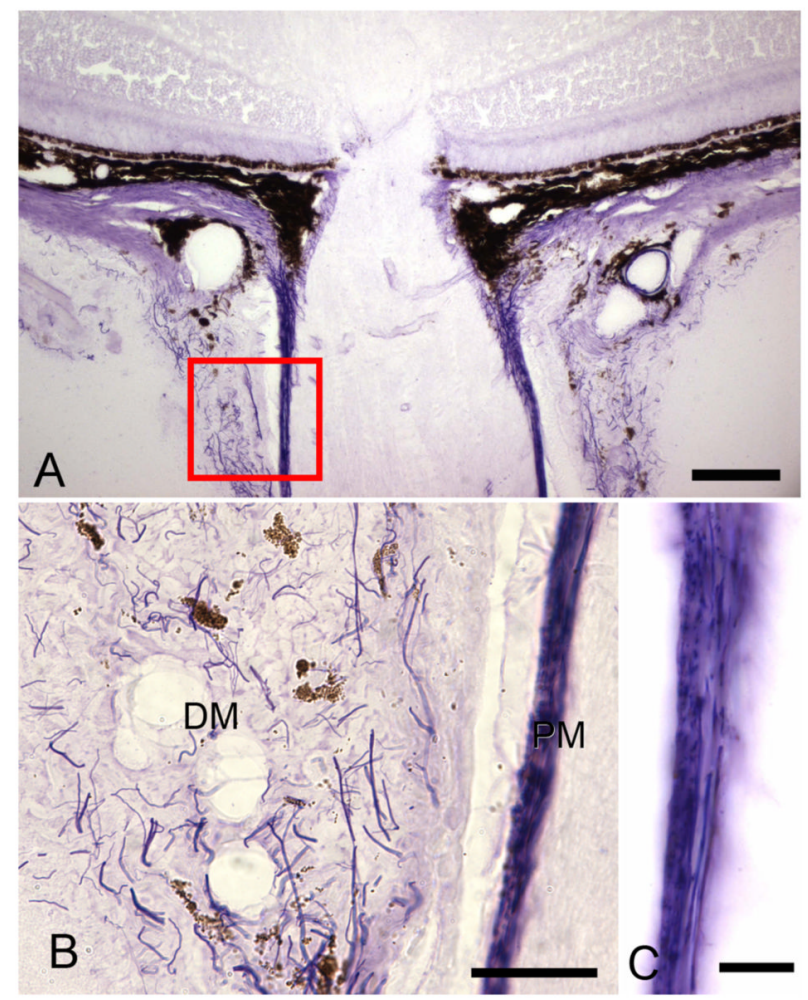

Figure 6.

The upper light micrograph (x20, bar=40 um) demonstrates the location of the magnified images (bottom) of Luna-stained elastin in the meninges. Elastin (arrows) is present in both the dura mater (DM) and pia mater (PM). B. Dura mater elastin shows no preferred orientation, with cross-sectioned fibers seen as dots or short segments and longitudinal fibers seen as extended lines $(x 63$, bar $=30 \mathrm{um})$. C. Pia mater elastin is arranged in two layers: an outer zone in which fibers run around the circumference of the nerve (seen here as dots) and an inner layer (seen as linear profiles) parallel to the long axis of the optic nerve $(\mathrm{ON})(\mathrm{x} 100$, bar $=10 \mathrm{um})$. 